\title{
Thoughts of an Immunobiologist about Covid-19
}

\author{
Tony Davies \\ Emeritus Professor, University of London, POLYREM Ltd, 81 Queens Road, Wimbledon, London, England
}

*Corresponding author: Tony Davies, Emeritus Professor, University of London, POLYREM Ltd, 81 Queens Road, Wimbledon, London, England; Tel: 442089447734; E-mail: tony.davies@blueyonder.co.uk

Received: May 16, 2020; Accepted: May 18, 2020; Published: May 25, 2020

\begin{abstract}
Attention is drawn to the existence of two very different facets of the immune processes operating consequent upon infection; Innate, a primitive mechanism which is quick acting and which plays a major part in inflammatory processes, and Adaptive, a mechanism that is slower to deliver elements specifically adapted from its recognition of the foreign invader. The cytokine storms that can be a harmful outcome of the response to infection derive initially from components of the innate immune system which, in addition to responding to foreignness, are activated by dead and/or dying cells of the infected host. It is suggested that although attack on the invading virus, by, say vaccination, seems the logical way to reduce the consequences of infection, it could be that exploration of the immunopathological effects of invasion could also help to specify means to reduce their impact. In particular it is suggested that prebiotics, orally ingested materials that can have beneficial effects on the gut micro biota, may be able beneficially to modify potentially harmful patterns of inflammation. In addition attention is drawn to the possibly exacerbating role of CRP an acute phase protein for which antagonists have been devised which could also help to reduce immunopathology.
\end{abstract}

The (London, UK) Times on05/04/2020 contained, on pp10-11, a double page spread by Tom Whipple, their Science Editor since 2011, on 'How Britain can beat Covid-19'. The useful table offered considers four approaches, Anti-viral drugs, Contact tracing, Herd Immunity and Vaccine (sic). Whilst not in any way wishing to dispute the utility of the facets of the approach presented, as an Immunobiologist for sixty years or so, I wonder whether we are missing a trick or two. It is widely recognized that all living things have interface mechanisms which regulate the interactions between themselves and the multiply complex aspects of the environment which impinge on their wellbeing. As human beings our thinking about these mechanisms is going to be tainted by what could be termed anthropocentrism and this can mislead us about the extent to which our thinking is germane to the totality of living organisms; i.e. is our thinking based on what pertains to Nature as whole? Perhaps most importantly we should try to make sure that our approaches to disease take cognizance of how living organisms in the wild state take care of themselves and how this is different from our own approach which is based on the advantages and constraints deriving largely from contemporary medical practice which can bear little relationship to recognition of the time taken for the evolution of the mechanisms which it deals with.

As humans we recognize five basic senses, sight, hearing, smell, taste and touch. Each of these has its own associated technology relating to the mechanisms involved and the various ways that they can go wrong and the defects corrected, as far as possible, within the existing framework of medical knowledge. All the senses involve complex patterns of neuronal stimulation and interpretation enabling us, often with training, to respond, as best we can, to such adverse environmental factors as, for example, excessive light, excessive noise, noxious gases, foul tastes and dangerously hot heat. We know either instinctively, or by learning, using aspects of our five senses, to avoid potential harm and what can either be pleasant or useful. Deployment of the five senses is similar in most vertebrates. In addition to the five basic senses, concerned directly with responses to environmental changes, there are internal more arcane senses which are also interface regulatory devices, operating either to protect us from danger or beneficially to enhance our life experiences. Some of these inner senses relate to such interactions as are involved with our responses to Covid-19 which we cannot see, smell, hear, taste or sense by touch. The inner senses are complex and include what are usually called the immune responses. The immune responses are widely believed, in a variety of ways, to be protective and to involve a non-cognitive learning element, referred to by many professional immunologists as immunologic memory. As far as protection is concerned this seems sensible in what can be seen as a hostile world that, following the thinking of Charles Darwin, Tennyson wrote of as 'Nature, red in tooth and claw'. The whole concept of 'immune' implies 'not affected by'. Wikipedia gives the meaning of 'immune' as 'resistant to a particular infection or toxin owing to the presence of specific antibodies or sensitized white blood cells. This definition could be thought largely to ignore one of the basic defense mechanisms of the body the innate immune response.

In fact the human body, and that of most other multi cellular animals, has a complex array of protective devices including the skin, which is a major albeit passive barrier to entry of potentially harmful agencies, the secretions of mucous membranes which can have antiseptic properties, the stomach acid which very effectively reduces the microbial content of our food and the gut microbiota (of which more later) that acts as a complex balanced ecosystem which is capable of resisting invasion by what could be called niche occupation, 
i.e. there is no room at the Inn. Underlying these various barriers to invasion which can be regarded as part of the immune apparatus are two complex reactive systems, the innate and the adaptive immune responses. The former involves a whole series of mechanisms the activation of which is particularly important in considering how humans react to invasion by Covid-19 and how we should use our knowledge of the mechanisms concerned to restrict as far possible the dangers such an invasion can lead to. The adaptive response is a slower process leading classically to the production of antibodies which are capable of binding specifically to the antigens which led to their production. The antibodies are seen as a specifically adaptive response to the antigenic stimulus and particularly important in relation to dealing with resistance to further invasions by the same organism.

Innate immunity is possessed by all triploblasts but only vertebrates have the capacity to enact adaptive immune responses. A recent article [1] gives a summary of the differences between innate and adaptive immunity. In outline, innate immunity operates immediately an appropriate stimulus is located. The cells operating the system, particularly macrophages, have significant capacity not only to recognize foreign entities but also, and very importantly in the present context of the background to viral infection, damaged and/ or dead cells of the responding organism. The target elements will usually be taken into the phagocytes where, in time, and with exercise of digestive enzymes they will be destroyed. Simplified portions of the phagocytically ingested material can be re-presented on the surfaces of the 'eating' cells for the attention of other reactive cells within the lymphoid system often initiating activity of the adaptive immune system. The phagocytes also, when they have been 'fed', emit a wide variety of secretions called cytokines. These agencies, of which their numbers are legion, can have powerful physiological effects which are important in considering what can be adverse consequences of invasion by Covid- 19 .

The assumption that all immune responses are primarily defensive has been questioned [2-4] The so-called Adaptive element, it is contended, far from being a device that always rids us of potentially dangerous pathogens by developing processes of rejection, has as a prime purpose active interaction with invading organisms, a virus, say, by reducing the viral burden to what can be a stable and potentially permanent accommodation which may well, in the long term, as far as the species concerned, be beneficial to invader and invaded alike. The benefits to the invaded are that resistance to further infection by the same organism is secured in addition to the acquisition of more potentially useful genes and their products. The benefit to the invader is that its survival is achieved which, had the host died, might not have been so likely. As the main author of the papers concerned wrote, 'the adaptive immune response, in many if not all instances can be, as its name suggests, thought of in natural circumstances as a 'Welcome mat rather than a Rejection slip'. Sadly during the process of accommodation, without medical intervention and often with it, some infected individuals may die. Later, the instance of myxomatosis infection of rabbits in Australia will be used to exemplify an extreme case of such mortality and how in some natural circumstances accommodation of host and invader can be achieved despite the existence of what is usually thought of as a mechanism always hostile to invaders.

The innate immune responses are not thought to be specific in that second contact with a specific stimulus elicits a better response than the first contact. The innate responses, in contrast to the adaptive immune response, are not recognized to have the specific memory that offers the possibility of making specific neutralizing antibody responses sometimes for many years. The innate immune response in evolutionary terms is one of the basic protective mechanisms with the added advantage that it also operates when for whatever reason dead or damaged cells can be mopped up, a kind of internal debridement process. The adaptive immune response characteristically operates by a system of antigen reactive $\mathrm{T}$ - and B-lymphocytes which are to be found throughout the body. As shown by the classical studies of the late Sir James Gowans, these cells have the capacity to escape from the main, high pressure, vascular system and recirculate via a low pressure lymphoid network that eventually feeds back into the main blood stream. The intricacies of the two systems involved in lymphocyte existence and behaviour were recounted in two texts $[5,6]$ which could be read with advantage by many contemporary immunologists.

Activation of both adaptive and innate immunity, particularly the latter, can have consequences for the general physiological status of the body in which the reaction takes place. These consequences often result in what is called inflammation a complex process that in extreme cases can lead to death. Two examples should suffice to indicate what can happen and why. Non-indigenous Zebu cattle in East Africa can suffer from East Coast Fever which is caused by Theileriaparva, a protozoan organism. Theileria has as its vector a species of cattle tick. When bitten by infected ticks T. parva can be transferred to cattle in tick saliva. In the infected host the protozoans can bind to and enter many types of nucleated (white) cells in the host blood. In the infected cells the parasite divides and can be liberated from the host cells into the blood stream where further infection of host cells occurs. Infected host cells which are activated and changed morphologically produce and liberate cytokines which are capable, inter alia, of drastically increasing capillary permeability. Within 14 or so days the infected animals may die in a most distressing way due in large part to loss of body fluids. This example of a lethal cytokine storm due to an infection is dramatic and it can affect almost all the organs of the body of the infected animal and, as will be argued, is comparable in some ways to what happens when influenza- like 'new' viruses such as Covid-19 kill humans. It should be noted, in the context of East Coast Fever, that some species of indigenous ungulates carry the parasite, ostensibly asymptomatically, in contrast to the non-indigenous cattle for which infection can be $100 \%$ lethal. In wild animals the host organism has somehow come to terms with the parasite and it is likely that these terms are determined by the manner of maintained activation of the total immunological apparatus.

As has been seen in the massive publicity associated with Covid-19, deaths from the influenza pandemic in1918 have also been attributed to cytokine storms. In the latest issue of Private Eye their medical correspondent, under the pseudonym MD, writes, in a version I hope he will forgive me for abbreviating:-'Severe Covid-19 
isn't an ordinary viral pneumonia. It's more of a dirty bomb, causing havoc in the immune and vascular systems. The overwhelming immune reactions can lead to a cytokine storm - death can be very quick. The havoc caused by the cytokine storm is due to the response of the body of the infected host rather than the virus itself which is not capable of cytokine production. Most individuals infected with the virus have minor symptoms perhaps also deriving from activation of elements of the immune systems but not with such drastic effects. The virus was probably the same with or without the cytokine storm and yet in two individuals that differ hugely in their response to infection we really cannot be sure what the different responses are due to. Clearly, we know some of the risk factors including age, weakened capacity to make immune responses, diabetes and a variety of other debilitating illnesses but what goes wrong to precipitate in some individuals such harmful responses as are occurring albeit more rarely than in younger and previously healthy individuals. Should we always be trying to kill the virus or should we be trying to intervene in the immunopathological processes which are a property of the host responding to the virus? This is not a new idea in that the elements of the inflammatory process have in recent years been the subjects of many investigations and it has been discovered that there are very many cytokines with a bewildering array of properties that can impinge in ways that are only just being discovered on the physiological well-being of individuals. The complexity of the system and the reductionism practiced by most research immunologists has perhaps helped to bring about the lack of incisive interventions in relation to cytokine storms. The Scientific Editor of the Times makes no mention of attempts to calm consequences of the immune response to the virus which can have, albeit relatively rarely, such disastrous consequences. Reduction of immunopathology is not yet part of the main stream thinking of many immunologists who are resolutely sticking to the adaptive elements of the immune systems with its specific antibodies, its specifically cytotoxic cells (usually displayed in vitro) and its apparent long term memory.

A long time ago Ian Clark, an Australian veterinarian, 'undertook research in innate immunity and disease pathogenesis at the MRC Clinical Research Centre at Harrow. It was during this period he began to develop the then novel view that infectious disease is caused not directly by the invading pathogen, but by the host's innate immunity to it. The host-derived mediators of this response, such as TNF (tumor necrosis factor) and IL-1, when produced excessively, were argued to generate disease through disrupting the normal homeostatic physiological processes. (from Clark's web site) On returning to Australia Clark continued to develop this idea that production of cytokines, particularly TNF is central to pathogenesis of non-infectious as well as infectious diseases'. Over several decades he and his colleagues carried out experiments the results of which show clearly that his early views in relation to the harmful role that could be played by innate immunity in infectious disease could be supported by strong evidence. Certain kinds of activation of the immune apparatus especially those which lead to production of TNF, one of the initiating elements in what may turn out to be a veritable cascade of cytokines that can lead to what is referred to as immunopathological damage. Such damage which is not due proximally to activity by the agents of infection but to massive stimulation of the elements particularly of the innate immune system. It could be thought of as an unintended side-effect. Clark showed in his work that TNF can play a vital role in triggering a harmful cytokine storm and that, if the capacity to produce TNF was variously suppressed, infected animals which would otherwise succumb to disease survived. He also showed that, if normal animals were injected with TNF, it was possible to mimic the manifestation of some of the symptoms infection in which it was supposed that immunopathological processes were active. Clark's work is seminal in beginning to understand how to reduce immunopathological damage.

Immunopathology attracts relatively little attention on the presently relevant web sites but the argument will be made here that its better recognition in conjunction with re-evaluation of the primary role of the adaptive immune response could add to our armamentarium of ways effectively to treat such dangerous epidemics as those caused by Covid-19. It is crucial to our understanding of how to treat Covid-19 infections to note that they are only rarely lethal but that, in serious cases when death seems likely, only symptomatic palliation presently seems possible. Why do some people die but the majority of infected individuals can suffer a relatively mild set of symptoms rather more serious than a common cold but not apparently much more dangerous?

The American Public Health Association has published for many years a manual entitled 'Control of Communicable Diseases'. This useful book, summarizes for many years for Public Health practitioners, what is known about all the infections that affect human beings and what can be done about them. It is a remarkable document from which much information can be gleaned about the generalities of infectious disease in Homo sapiens. The following points are germane to the present arguments.

1. There are millions of species of microorganisms, Viral, Archaean and Bacterial of which only two hundred are regularly harmful to humans; the vast numbers of remaining organisms are ostensibly of as little significance to us as we are to them. The idea that the micro-organismal hordes are lurking to attack us is not supported by the facts of what is to be found in the big wide world of living organisms.

2. Very many of the organisms recognized as infectious are known to exist asymptomatically in human hosts. For example in relation to de novo contact with Mycobacterium tuberculosis it is authoritatively stated [7] 'Approximately 90$95 \%$ of those initially infected enter a latent phase from which there is a lifelong risk of reactivation'. This in relation to what is commonly accepted to be causing globally the biggest annual human lethality from an infection. What do we know about the immunological status of the $90-95 \%$ of infected humans who normally develop no symptoms? Relatively little, perhaps on grounds that if it is not apparently broken we do not need to know how to repair it. What do we know is the immune status of those infected with Covid-19 virus but with minor symptoms or none at all? Is contagion possible from infected individuals with few or no symptoms? Does the virus live on after the signs of acute infection have disappeared? 
3. Some pathogenic viruses can successfully be 'vaccinated' against using living but variously attenuated, viruses. The way that Henderson, working with WHO, led the campaign to eradicate small pox as a disease by prophylactic vaccination using live cow pox virus exemplifies what can be done. It should however be noted that the principle involved had been discovered by Jenner two hundred years previously and that Henderson, working in the WHO, with a massive global campaign found :-'Vaccination brought some control, but the key strategy was "surveillance-containment". These techniques entailed rapid reporting of cases from all health units and prompt vaccination of household members and close contacts of confirmed cases'.

It took ten years successfully to eradicate small-pox by creating the circumstances in which the virus could no longer at the time find a susceptible host. Whether a few decades later the lack of susceptible hosts would still pertain is an interesting question which, let us hope, will never be answered. In the present context the necessity of surveillance containment should be noted as a likely requirement for a fully successful vaccination campaign against Covid-19.The contemporary injunction from the head of WHO to test, test, and test is compatible with this notion.

\section{In relation to Covid-19 it seems that}

1. It is common that infectious organisms, called infectious because of the damage they can cause, often do not cause much if any damage. Those infected with Covid-19 who remain without symptoms or with minor illnesses, that is a large majority, exemplify the genre. However it should be noted that for a highly contagious virus, which can kill $1 \%$ of those who catch it, there are serious public health problems in dealing quickly with $1 \%$ of a very large number of infected individuals in a relatively short time. As the antagonists of Covid-19 point out this could be enough to overwhelm Health Services if they were not adequately prepared.

- Vaccination is a possible means to avoid the damage caused by Covid-19 but it could take a longish time to enact a successful campaign This is not to say that the strenuous efforts to produce a vaccine for Covid-19 should not continue but there should be a note of caution in the time it might take in addition to the requirement for global surveillance and containment.

2. Some anti-viral vaccination campaigns, successful over long periods of time (yellow fever virus for example), have used live viruses. Immunization against influenza symptom causing viruses have not so far involved live viruses but fragments with what are thought or known to be the important elements of viral protein reaction to which can lead to protective antibody production. The issue of whether immunological memory supposedly in due to the adaptive immune system, is active here is important but it is possible that the enormous genetic potential of the gut microbiota, with at least 100 times as many genes as on the human genome, could provide a huge array of antigens from which there could be a trickle of the specific antigenic material cross reactive with non-living viral antigens used to resistance to infection. Such material could help to maintain a low level of active immunity once an initial vaccination with a non-living agent has occurred. Is it possible that the human microbiome could be changed a little to ensure the relevant specific trickle could be created without persistence of a living element in the vaccine? Alternatively if a living virus is used as the active agent in a new vaccine are we trying to arrive at accommodation or outright rejection? If the latter, as shown for a number of infections there is, after a short time, no residual resistance to re-infection. Immunological memory is perhaps a variable feast.

The almost total lethality of rabbits in Australia deliberately exposed to Myxomatosis virus in the 1950s indicates what can happen. Some twenty five years later it could be supposed that the remaining quite numerous rabbits were resistant to, immune, to the virus and this is certainly a possibility but it emerged that the virus had anyway, in the intervening period, now got reduced pathogenicity; whereas previously it had killed the overwhelming proportion of infected rabbits it now killed only a quarter of newly infected animals.

Contemporary humans, encouraged by advertisements from the manufacturers of antiseptics, are often totally xenophobic in relation to microbiological organisms but this view may change. It is recognized that humans, along with other multicellular animals that have an alimentary canal, have associated microbiomes of microorganisms in and on their bodies. As far as humans are concerned the number of cells of micro-organisms carried by the human body is of the same order as the number of human cells (tens of trillions). Whether we like it or not we are, like most if not all multi cellular organisms, extraordinarily complex collections of many species of organism. There can be little doubt that our association with the microbiome represents symbiosis; living together for mutual benefit. Two recent texts $[8,9]$, written for the general public, detail this state of affairs. The more daunting thing is how to reconcile these clear facts with the, seemingly xenophobic, concept of immunity. It should also be noted, in the present context, that it is likely that the microbiome has a major role to play in the maintenance of normal health. ISAPP, the International Scientific Association for Pro- and Pre-biotics has put an authoritative document online [10]. Giving the details of this state of affairs.

It is not presently known how the microbiome affects body physiology but it seems likely that it does so in part by affecting the brain impinging on the hypothalamic pituitary adrenal axis, HPA, which in turn can have an impact on the endocrine functions in the body. A recent book entitled The Psychobiotic Revolution, Mood, food and the New Science of the Gut-Brain Connection [11] gives a flavour of how this line of thought is being developed. How the gut flora affects the brain is not known with certainty. It has been suggested, and there is supportive evidence for this idea, that cytokines deriving from the innate immune apparatus, perhaps from the Kupffer cells of the liver stimulated by the continuous flow of material from the gut microbiota into the liver via the hepatic portal vein, are important. The stimulated liver sends the cytokine messages via the vagus nerves to 
the brain [12]. In recent work kindly undertaken by Prof Gareth Leng who, though not deeming the results adequate for general publication, has no objection to it being written about, established that, in rats within a short time of oral ingestion of a prebiotic, there were clear indications of neuronal activation [13] in the paraventricular nucleus, a component of the hypothalamus. Prebiotics cannot by definition be absorbed from the gut into the body proper but, again by definition, they have a beneficial effect on the gut microbiota. This useful finding could indicate part of the route along which the brain can be affected by gut activity.

Significant reduction of inflammation, induced in the colon of rats by trinitrobenzenesulfonic acid, was obtained by oral feeding with a prebiotic incorporated in the chow fed to the animals [14]. In addition, in the treated animals four clinical biochemical markers of inflammation, TNF, Il-1, IL-6 and myeloperoxidase activity (this latter a marker of neutrophil activity) were significantly reduced. This demonstration shows that prebiotics can have a significant prophylactic influence on inflammation. The experimental system adopted, pioneered by Morris et al [15], is widely used to explore ways of treating colonic inflammation. In the recent study discovered changes in the gut flora, concomitant with the use of the prebiotic, are an interesting correlate. It was also shown that the prebiotic use was, in terms of reduction of inflammation, better than sulfasalazine or infliximab, both standard treatments in man for reduction of colonic inflammation. We are only just beginning to come to know enough about inflammation to look towards having available more evidencebased control by methods other than administration of so-called antiinflammatory agents such as steroids. These can certainly be effective but they can have undesirable side effects and anyway are not given on the basis of a firm understanding of inflammatory processes. It is significant that there are cytokines which are known to be antiinflammatory and others pro- inflammatory. For example, the Kupffer cells in the liver and macrophages in general, the significant elements in the array of cells involved in creating innate immune reactions[16], are sometimes liberators of pro-inflammatory cytokines and sometimes liberators of anti-inflammatory cytokines. Whether these two different cells are ontogenetically distinct or whether according to the prevailing circumstances will produce one or other of the kinds of cytokine seems not to be known. It can be queried why do we need both forms of activity?

Reference to the process of wound healing could give a simple indication of the separate positive roles of pro- and anti- inflammatory influences. Consider a small wound inflicted by, say, a knife on the back of a human hand. There is an elaborate mechanism for stopping the blood flow. Once this has been achieved some of the cells liberated from the blood stream become active in tidying up the wound in a manner that could be termed pro-inflammatory. Visually for a short time the wound could look inflamed but all being well within a few days the healing process starts to occur and it likely during this time that anti-inflammatory influences are active to help promote cell movement and the cell division of the local epithelia required to bring about a full repair. The two elements of inflammation in this way are complementary in leading to what superficially seems to be an uneventful wound healing. The balance between pro- and anti- inflammatory influences will normally work sequentially in such a way as to help to maintain body integrity but if the balance is disturbed potentially harmful pathological changes can be an outcome. For the moment we are not sure how generally to maintain the requisite order of change from pro-to anti- inflammatory processes or to redress imbalance but it seems possible the microbiome could be influential in this respect.

The acute phase response is a highly conserved, cytokine mediated, non-specific response to almost all forms of tissue injury, infection and inflammation. A key component is the sensitive, rapid and dramatic increase in the circulating concentration of the so-called acute phase proteins. The classical human acute phase protein is C-reactive protein (CRP), the plasma concentration of which closely corresponds with the presence, extent and activity of most diseases and their response to treatment [17].Indeed, the universal, routine measurement of CRP concentration is one of the most widely used clinical chemistry assays. CRP values are also importantly prognostic in many conditions, including Covid-19, consistent with CRP not merely reflecting disease activity but also acting as a pathogenic factor, as first demonstrated by Pepys [18]. CRP binds specifically to dead and damaged cells in vivo and then activates the powerfully proinflammatory complement system, thereby exacerbating pre-existing tissue damage. The original small molecule CRP inhibitor designed by Pepys [19] reduces the morbidity and mortality of severe influenza A virus infection in a mouse model [20] (4) and his subsequent more potent inhibitor is now in full scale development for clinical testing in Covid-19 and other indications.

Contemporary medical practice has enabled increase in the average expectation of human life in what we call the Western World from about 40 years of age in the middle of the nineteenth century to its present 80 or so level. We have also learned how to repair or replace at least some defective body organs and thus prolonging the lives of those who in years gone by would have died much earlier. Human expectation now is that when life can be preserved it should be. In so-called wild populations of animals such artificial preservation cannot occur and life span is determined by whatever living circumstances constitute the environment. Prolonging human life span has advantages and is now an expectation but perhaps it should be recognized more widely that there are demographic down sides in terms of building up more elderly humans the medical requirements of whom are more onerous and expensive than those who created the remarkable NHS anticipated.

\section{An old immunobiologist, suggests}

1. We should recognize more clearly that Homo sapiens, as is true for most other living organisms, is not just a single species but an extremely complex collection of organisms with most of which for most of the time we live in harmony. We have within us what is known as a balanced ecosystem of microorganisms that we are largely unaware of. Improving our recognition of what is a clear fact could help in time to maintain human health. We should learn how better to look after our inner microbial garden. Learning more accurately to manage our microbiomes could help to restore the balance between pro- 
and anti- inflammatory influences disturbance of which leads to the often painful diseases with immunopathological component from which humans and their domestic, farm and companion, animals can suffer.

2. Infectious disease can be seen as, naturally, an evocation of a slow evolutionary process to accommodate more organisms some of which, in time, will be moved from the status of parasites or commensals to slaves contributing freely to our welfare. Such a vision is not comfortable for those dying as consequence of contact with an apparently new organism encountered for the first time. By way of consolation, it should be better known that nearly all the nucleated cells in the human body contain mitochondria. These self-replicating organelles, containing their own DNA, many millions of years ago were free living bacteria. Aggregation of such organisms, followed by genetic simplification involving loss of genes made redundant during division of labour, left the current mitochondria which essentially manage energy supply in human cells. In plants the chloroplasts which are capable of collecting the energy from sunlight and using it to manufacture energy containing molecules the break- down of which we rely on in big scheme of things to make things happen are, like mitochondria, of archaean/ bacterial origin. We are totally dependent on the activities of genetic material derived from other species of organism.

3. Prebiotics could play a role at least prophylactically and possibly therapeutically in helping to reduce the morbidity of Covid-19 infections by beneficially affecting the gut microbiota which in turn is capable of influencing the pattern of immunopathological changes that can lead to a dangerous cytokine storm.

4. Antagonists of CRP could be used to help to inhibit the cytokine storm which is associated with the lethality of Covid-19 infection.

5. The Science Editor of the Times did a good job helping us to follow the dictates of our political masters who in turn claim; somewhat uneasily it has to be written, to be led by scientists. The scientists concerned not surprisingly adopt the establishment views of what is important but it can seem to other scientists that the views being used to determine action in the Covid-19 pandemic are lacking in imagination particularly in not trying to diminish the immunopathological response of a few of those infected and being able to predict which ones are most likely to suffer.

\section{Acknowledgements}

I am grateful to my colleagues Dr Attila Erdos and Dr Deirdre McIntosh for their careful reading of and comments on the manuscript
I would also like to thank Dr Chris Owens a good friend for his intelligent interest and helpful suggestions. To Prof Bruce Reid, an erstwhile colleague and good friend, I am most grateful for the time he took over the manuscript and his incisive and useful comments. The support of my wife, Agneta, for her unswerving backing at all times was invaluable. Professor Sir Mark Pepys kindly drewmy attention to the significant role that CRP could be playing in Covid-19 infections and his creation of a likely solution to one of the problems thereby created.

\section{References}

1. Sagar Aryal (2018) Difference between Innate and Adaptive Immunity. Microbiology Info.com.

2. Davies AJS, Hall JG, Targett GAT, Murray M (1980) The biological significance of the immune response with special reference to parasites and cancer. J.Parasitol 66: 705-721. [Crossref]

3. Davies AJS (2008) Immunological Tolerance and the Autoimmune Response. Autoimmunity Reviews 7: 538-544.

4. Davies AJS (2012) Immigration control in the vertebrate body with special reference to chimerism. Chimerism 3: 1-8. [Crossref]

5. Principles of Bacteriology and Immunity (1964) Topley and Wilson, Fifth Ed, Edward Arnold.

6. Lymphatics, Lymph and the Lymphomyeloid Complex (1970) Academic Press.

7. APHA Control of Communicable Diseases Manual 17 $7^{\text {th }}$ Edition, 2000: 521.

8. The Diet Myth, The Real Science behind what we Eat. Tim Spector (2016) Wiedenfeld and Nicholson.

9. I contain Multitudes (2016) The microbes within us and a grander viewof life. Ed Yong, Vintage.

10. (2017)Nature Reviews Gastroenterology \& Hepatology. 14: 491-502.

11. The Psychobiotic Revolution, Mood, Food, and the new Science of the Gut Brain Connection (2017) SC Anderson, JF Cryan, Ted Dinan (eds.). National Geographic, Washington.

12. Natasha Corbitt, Shoko Kimura,y KumikoIsse, Susan Specht, Lisa Chedwick, et al. (2013) Gut Bacteria Drive Kupffer Cell Expansion via MAMP-Mediated ICAM1 Induction on Sinusoidal Endothelium and Influence Preservation-Reperfusion Injury after Orthotopic Liver Transplantation, The American Journal of Pathology 182:180-191. [Crossref]

13. Prof Gareth Leng, Personal Communication.

14. AVIDIN Kft, Szeged. Hungary,Contract Research ,Polyrem Study, 2019.

15. Morris GP, Beck PL, Herridge MS, Depew WT, Szewczuk MR, et al. (1989) Hapten-induced model of chronic inflammation and ulceration in the rat colon. Gastroenterology 96: 795-803. [Crossref]

16. https://www.cusabio.com/c-20938.html, M1 and M2 Macrophages

17. Pepys MB, Hirschfield GM (2003) C-reactive protein: a critical update. J Clin Invest111:1805-1812. [Crossref]

18. Griselli M, Herbert J, Hutchinson WL, Taylor KM, Sohail M, et al. (1999) C-reactive protein and complement are important mediators of tissue damage in acute myocardial infarction. J Exp Med 190:1733-1739. [Crossref]

19. Pepys MB, Hirschfield GM, Tennent GA, Gallimore JR, Kahan MC, et al. (2006) Targeting C-reactive protein for the treatment of cardiovascular disease. Nature 440:1217-1221. [Crossref]

20. Gao R, Wang L, Bai T, Zhang Y, Bo H, et al. (2017) C-reactive protein mediating immunopathological lesions: a potential treatment option for severe influenza A diseases. EBioMedicine 22:133-42. [Crossref]

\section{Citation:}

